\title{
The Art of Translation: On Translating Sukumar Ray's Abol Tabol in English
}

UMA RAY SRINIVASAN

\begin{abstract}
This essay is primarily an attempt to lay out the intricacies of the process that have been experienced while translating in English what is perhaps the best-known nonsense verse of our country, Abol Tabol (containing fifty-three poems) by Sukumar Ray written in Bengali. This analytical process also reflects upon similar difficulties one may encounter in translating nonsense verse in general. The short Part I of the essay considers the challenges of translation, which critics have always pronounced and which has been experienced by this writer. Part II specifically discusses how these challenges are encountered in translating Abol Tabol. It considers the linguistic eccentricities of Bengali that make the translator's task difficult, but also rewarding; and it also considers other nuances of verse translations such as the use of rhyme, rhythm, vocabulary, proverbs and idiomatic expressions, word coining, etc. each of which is assessed especially in the context of Abol Tabol translation. This article also presents a few samples of individual pieces of translated poems by this author wherever they help amplifying the points discussed.
\end{abstract}

Keywords: Translation, Language, Rhyme, Rhythm, Sukumar Ray.

\section{Part I: Preliminary}

\section{The Act of Translation}

Saussure, among others, foregrounds the untranslatable nature of a literary text in a given language, which is a product of a certain cultural and temporally circumscribed entity. For language is not merely a naming system, but a system of 
carrying and communicating cultural values. Since different cultural groups think about the world in different ways what is expressed in one cultural context may not be axiomatic in another context. Language thus has an important role in 'realizing reality' (Kate McGowan, 'Structuralism and Semiotics,' The Routledge Companion to Critical Theory, 5). This poses a serious difficulty in all kinds of translation exercises. In McGowan's words, 'If you speak more than one language, you will already be familiar with the impossibilities of translating conceptually from one language to another' (ibid. 5). A translator's anxiety about authenticity is, therefore, a very real issue, impeding her very act of translation.

Abu Said Ayub, in his Introduction to his remarkable prose translation of Galib's Gazals, expresses this anxiety when he admits that, even a reasonably 'good verse translation' is bound to diverge from the original (10), so his Bengali translation of the Gazals is not attempted in verse. In this context Ayub mentions the notorious instance of Rabindranath Tagore's grossly inadequate translation of John Donne's lines, 'For god's sake hold your tongue and let me love' - 'দোহাই তোদের একটুকু চুপ কর,/ ভালবাসিবারে দে আমারে অবসর।' ('Dohai toder, ektuku chup kor, / Bhalobasibare de amare abosor'). Ayub, however, says in the same context that he has read the very best translation also in Tagore (11). Recalling the paradigmatic failure set before us by Ayub may not be the most encouraging beginning for someone set to explore the prospect of translating Sukumar Ray's Abol Tabol. But it is a useful or even a necessary caveat that a translator might bear in mind. Taking up a piece of text and translating it in another socio-cultural-lingual sector should not be allowed to end up as an uncalled for transmutation of the text which to others might appear an unwarranted distortion. 
The Art of Translation: On Translating Sukumar Ray's ...

And yet, the best ideas expressed by a writer in her own language would remain confined to her own lingual group and the text would remain just 'a symbol, with mystical or magical properties in itself,' and not 'a messenger conveying meaning' as Fiona Sampson says in her essay 'Creative Translation,' The Cambridge Companion to Creative Writing (121). So it has to be translated and transmitted in other languages to make it accessible to all. This is the rationale behind all translations. According to Willis Barnstone, 'The act of translation is the other Babel, that impossible tower,' that is needed to break the 'solitude of difference' (3). The translation act is this precise aqueduct, though artificial, yet necessary, for conveyance of ideas from one side to the other, connecting the different peoples of the world.

Translation, therefore, is a worthy exercise, a necessary exercise. Yet this desirable exercise perhaps is one of the most challenging one, for there are two sides to all translation works: it has to meet the standard of authenticity of the original work while it has to be authentic to the requirement of the language in which it is translated. A translation in the first place is naturally expected to be an honest representation of the original. A mirror is expected to produce an exact reflection of the object placed in front of it. The better the quality of the mirror the better the impression it produces. But still the mirror metaphor is not wholly satisfactory. The translation work is not as mechanical a process as placing an object before a mirror intended to reflect it. A translation which attempts a very close representation of the original work, literally seeking to present it in another medium might fail to bring it alive in the medium in which it is presented. The demands of the new medium must be addressed, too. Being authentic without being stiff, being accurate, without being mechanical, and getting the content, without missing the spirit is a feat which is difficult to achieve. This takes us to the question of the difference between 
the verbatim translation of a piece and translating its innate spirit. The former is expected, or, even necessary, when legal or business matters and matters of subjects like science are translated into another tongue; this may not, however, be the ideal mode to be practised when it comes to translating a literary piece. Maintaining a proper balance between the two is the achievement of the best translation when we deal with literary texts.

It is necessary to remember that translation is the best translation that does not read like one. To make this possible some liberties must be granted of course. The permissibility of the liberties taken by the translator will naturally depend on the factor that no outrageous changes are wrought to distort the content and essence of the original. In other words, a translation should better not turn itself into a transgression. The translator who bears these in mind is the most successful translator. Explaining the efficacy of Shelley's translations of some of the masterpieces in Greek, Latin, Italian, Spanish, German, and French R. B. Woodings says in his 'Editor's Note' on Joseph Raben's 'Shelley as Translator,' that Shelley in these works was 'not merely concerned to find verbal equivalents for the original, but also sought to express the dramas as he understood them' (196). These problematic aspects of translation activity have been borne in mind by me in seeking to translate Sukumar Ray's Abol Tabol and which I have laid down in Part II of this discussion.

\section{Part II}

\section{Translating Sukumar Ray}

'The vanity of translation' should, therefore, give way to the fullest appreciation of the sensitive nature of the text which is delicate as 'violet' and its 'colour' and 'odour' are as easily 
lost in the process of translation, as Shelley warns us in his $A$ Defence of Poetry (7). Translation of a verse piece necessarily has this additional challenge of negotiating the subtle nuances of the medium such as metre, rhyme, rhythm, texture of the words used, the pace and such other unique poetic features. The famous instance of the radical, yet unintentional change that underwent in the process of translating and recreating the Petrarchan sonnet in English in the Renaissance England may be recalled. The felicity of rhyming words in Italian language was countered by the paucity of rhyming words in English language - a serious factor that left its indelible mark in the three decades of sonneteering history in England - 1580s to 1600. Writing a fourteen-line poem in a matter of five-rhymepattern for an entire sonnet cycle comprising a hundred sonnets, proved more of a task in English than in Italian and the former converted the latter to a seven-rhyme-structure giving birth to the English sonnet structure ending in its powerful epigrammatic couplet. The couplet ending in the sonnet structure is regarded, however, as an extraordinary stroke of serendipity than anything else, something to which we owe the rich treasure-house of sonnets of Shakespeare and his contemporaries. This once again confirms, as Fiona Sampson says, the 'linguistic resources which make rhyme easier, (more organic) or more difficult' differ in different languages (Sampson, 122). What this note emphasizes is that felicity of two languages differs considerably - what is expressed in one language may not be matched exactly in the same terms in another language.

And in undertaking the translation act if one has the misfortune of not being included in Eliot's category of 'good poets' one should at least try to have the consolation of not being

${ }^{1}$ The English language, however, we all know, contains the very best sonnets in Petrarchan form written later. 
relegated to his category of 'immature poets' and 'bad poets' (125).

\section{2}

The difficulties of translation exercises, discussed in the previous section of this essay, are magnified many times over when it comes to translating nonsense verse. In translating nonsense rhymes the translator's job is complicated by multiple factors: a) Nonsense rhyme is born in and is nourished by the specific scope of an individual cultural lingual group. b) Consequently, the sense, or paradoxically, the non-sense, which goes to build a specific body of non-sense rhyme may in all likelihood generate no response in another alien set up. c) This genre of literature, more than any other, frequently uses idiomatic expressions and proverbial sayings on which the very point of the fun in the context depends, which is difficult, and perhaps even impossible, to translate ${ }^{2}$ d) Nonsense rhyme, in any language, is sure to generate its own breed of nonsense words either already existing in the language, or freshly manufactured by the writer to fulfil the need of the immediate context. While the original creation is already an acclaimed and accepted masterpiece, there is the danger of anyone challenging the legitimacy of these nonsense words generated by the translator. ${ }^{3}$ e) And finally, the success of a nonsense rhyme depends on its content and its spirit of fun that is intricately connected to rhyme, rhythm, metre, vocabulary, sound pattern and pace all of which comes as a single package, and to get the entire composite package

2 The noted scholar, Sukanta Chaudhuri mentions this point in his 'Translator's Preface' in his translation of Sukumar Ray's Abol Tabol, The Select Nonsense of Sukumar Ray.

${ }^{3}$ Nirmal Gupta, in his Preface to his translation of Sukumar Ray says: 'Whereas nonsense writers have the liberty to freely coin words giving sonic and imaginary effects, a translator has not,' 5 . 
translated into another medium is a task, that in all probability, is doomed in its very inception. Sukanta Chaudhuri mentions this difficulty in his essay, 'Sukumar Rayer Anubad,' ('Translation of Sukumar Ray') Sukumar Parikrama (142) . Pratap Chandra Chunder states: 'To translate an ordinary poem or verse into another language is itself a difficult task. More difficult is to translate a nonsense verse or satirical story, as one has to retain the sense of the original nonsense or satire in translation.... But a nonsense verse loses much of its charm if the original lilt, jingle and play on words are absent in translation. To be appealing nonsense verse must stick to rhyme but not reason. To a translator this is another difficult hurdle (Foreword, Whymsy Wave, Nirmal Gupta). For the texture and typicality of a language is so unique that a simulacrum of the exact texture may be defied by those of another language. Just as science affirms that water boils at different temperature levels at different altitudes, similarly the fun evoking or fun provoking elements and components may not be the same in two or more different mediums.

Since the nonsense rhyme defies sense one cannot translate it into another medium - for only a matter that conveys sense could be translated, if at all. So what one may at best attempt is not translating the sense (or, the nonsense), but transporting the spirit of a set of nonsense rhymes in another language. This has already been pointed out in Part I of this essay and now we may demonstrate it by citing what may be taken to be the best

\footnotetext{
4 As Sukanta Chaudhuri points out, it is not a translator's primary responsibility to meet the reader's expectation that a specific word or expression in the original be transported in the translated medium. The translator has to bear in mind the overall impact of the passage - its rhyme, rhythm, speed, the syntactical structure and its phonetic impact - and in this activity the translation of individual word constructs no more than one step taken in that direction. 'Sukumar Rayer Anubad,' Sukumar Parikrama (142).
} 
instance of translation of a nonsense verse in modern Bengali - Satyajit Ray's তোড়ায় বাঁধা ঘোড়ার ডিম (Torai Bandha Ghorar Deem, i.e. A Bouquet of Horse's Eggs) where Ray never claims to have 'translated' either Edward Lear or Lewis Carroll; but, he says in his brief introductory to the book that his non-sense rhymes are inspired by the masters (7). Thus we find that his Bengali limericks are his own adaptations and recreations of their works, not translations. In some instances he re-creates from the ideas suggested by Edward Lear's drawings. Some of Ray's translations ${ }^{5}$, one may even venture to say, adds to the fun of the original; they are not mere translations in this sense. We may take for example, Lear's

There was an Old Man who supposed

That the street door was partially closed;

But some very large rats,

Ate his coats and his hats

While that futile old gentleman dozed. ${ }^{6}$

This limerick gets a new twist in Ray's

চোরের ভয়ে রামনারায়ণ থোট্টা

মুমোয় ব’সে বন্ধ ক' রে দোরটা,

এই সুযোগে দুই ইঁঁুরে

দিব্যি থেলো পেটটি পুরে

ঝোলানো তার সাধের হ্যাট আর কোটটা।

Chorer Bhoye Ramnarayan Khotta

Ghumoi bose bondho kore dorta,

Ei sujoge dui indure

Dibbi khelo pet ti pure

Jholano tar sadher hat ar coat ta.

${ }^{5}$ All Ray translations of Lear are cited from Toray Bandha Ghorar Deem.

${ }^{6}$ Parrott, 25. 
Again, we may take the piece, 'There was an old man with his owl,' which has been entirely recreated by Ray in his 'কে জানে এ নিশাচর দেথে মোরে কি চোথে,' ('Ke Jane E Nishachor Dekhe More Ki Chokhe') where the vestige of the original is not found anywhere except in Lear's drawing. We may place the pieces side by side:

There was an Old Man with an owl, Who continued to bother and howl;

He sat on a rail

And imbibed bitter ale,

Which refreshed that Old Man and his owl. ${ }^{7}$

'কে জানে এ নিশাচর দেথে মোরে কি চোথে!'

মোর পাশে দেথে যদি ভাবে অবিবেচকে

এ আমার কেউ হয়,

আমি বলি মোটে নয় -

কোনোখানে মিল নেই মানুফে ও পেচকে।'

'Ke jane e nishachor dekhe more ki chokhe.

Mor pashe dekhe Jodi bhabe abibechoke

E amar keu hoi,

Ami boli mote noi-

Konokhane mil nei manushe o pechoke.'

In fact, কোনোখানে মিল নেই, রায়ে ও লিয়রে! (konokhane meel nei Ray-e o Lear-e), that is to say, Ray clear sweeps past Lear!

${ }^{7}$ Lear, http://lear200.com/there-was-old-man-owl 
From this we may conclude that a literal translation of a nonsense verse is neither desirable, nor possible.

\section{3}

We may now consider some of the unique difficulties encountered in translating Abol Tabol. There are specifically at least four kinds of difficulty one encounters in translating $\mathrm{Abol}$ Tabol owing to the essential difference between the syntactical practices (alone) of the two languages, the uniqueness of English and Bengali: (a) Bengali is devoid of the accessories like articles - definite and indefinite articles. Hence when a given content is translated from Bengali to English the inclusion of these articles in English, a necessary grammatical and structural requisite of the language, adds to the extra syllables making the expressions wordier. This, in fact, is one of the factors that affect the characteristic metrical propensity of the two languages: while the falling rhythm of trochees and dactyls is more spontaneously practised in Bengali, the opposite is true of the English verse, which is prone to take to rising rhythms of iambs and anapaests. ${ }^{8}$ This point is taken up again in this article in discussing the metrical pattern of Sukumar Ray and its translation in English. (b) In Bengali, the practice of using a single syllabic unit as a suffix at the end of words enables one to use less syllables in conveying more meaning. In English at the least a preposition must serve the purpose. For instance গায়স (payash) becomes গায়সে (payashe); আফিস (office) is changed to আফিসে (office-e); কালচুল (kalochul) could be কালচুলে (kalochul-e); আমিয (amish) is changed to আমিযেতে (amishet-e); বর্ণনা (barnana)is converted to বর্ণীতে (barnit-e); সাবান (saban) can be সাবানের (sabaner);

8 See Sukanta Chaudhuri on this point in 'Sukumar Rayer Anubad,' Sukumar Parikrama, 144. 
The Art of Translation: On Translating Sukumar Ray's ...

বালিশ (balish) can be changed to বালিশে (balish-e); দেয়াল (dewal) can be দেয়ালে (dewal-e) ${ }^{9}$. All these are instances taken from the single poem 'ট্যাঁস গরু,', 'Wow Cow'. Yet if we seek a parallel English expression it requires a whole new word to be tagged. For example, we may take the last of the word mentioned here from 'ট্যাঁস গরু,' 'Wow Cow,'- 'দেয়ালে' ('dewale') in the phrase 'ঠ্যাস দিয়ে দেয়ালে' ('thyesh diye dewale') which has to be translated as 'lean against the wall' (used by me); 'crouched by the wall' used by Sukanta Chaudhuri, The Select Nonsense of Sukumar Ray, 41; 'propped up against the wall' by Niladri Roy, 48; 'resting weary against a wall' by Sampurna Chattarji, 40 (italics in cited lines are mine). (c) Onomatopoeic words are far greater in number in Bengali and many other Indian languages than in English and which have been profusely used by Sukumar Ray for their funevoking quality. For instance, if we consider 'ট্যাঁস গরু,' 'Wow Cow' again, we find the following words: 'ফিটফাট্' (fit fat), 'লটথটে' (lot khot-e); 'থটথট্' (khot khot); ‘ল্যাগব্যাগ়' (lag bag); ‘খকথক্' (khok khok); ‘ঘিনঘিন্’ (ghin ghin); and ‘ঠক ঠক’ (thok thok). (d) There is a technical snag, to use Fiona Sampson's words, 'English is not derived from a single root, then garnished with loanwords. Because it's split at the root by Germanic and Romance origins, it's relatively difficult to unify with matching sounds.' (123).

\footnotetext{
9 'গায়স' (payash) is a sweet dish the chief ingredients most commonly used are a special variety of fine rice and milk, 'আফিস' is the Bengali version of 'office'; কালচুল (kalochul)stands for 'black hair'; আমিয (amish)refers to all kinds of non-vegetarian dishes; বর্ণনা (barnana)stands for 'description'; সাবান (saban)is 'soap'; বালিশ (balish) means 'pillow'; দেয়াল (dewal) stands for 'wall'.
} 
I cite my translation of Sukumar Ray's poem here, which will fully amplify my point:

\section{ট্যাঁস গরু (Tyansh Goru) The Wow Cow}

The wow-cow is not a cow, but it's a bird, In Haru's office is it seen, I've heard.

Its eyes are dozy, but its face is flossy.

Its hair is done in a bun so stylish 'nd glossy.

This horny creature's tail is twisted 'nd crooked.

You only touch it — lo! a horrid racket!

A pathetic rachitic creature, bony ' $n$ ' rickety,

It'll tumble down if only you flick at it.

My feeble words, alas, do fail to describe it,

This drawing is the only means to depict it. ${ }^{10}$

The wow-cow like other cows will not say moo,

It leans against a wall and says boohoo!

At once it yells and shouts and makes much fuss,

The reason though for doing so one can't guess.

It dislikes green grass and peas, or hay and fodder,

The sight of other food can make it dodder.

To slurp soap soup, to lick a candlestick,

Is its modest pick which, if refused, it falls sick.

It shakes and coughs on any food substitution.

You see it has a sensitive constitution.

Once when it tried to eat a piece of rag,

It fell into such a lag; its spirit did sag,

And for three full months it remained half-dead in bed.

A creature prized for its features, so they said.

The wow-cow is indeed a truly worthy pet,

You may come purchase it at a discounted rate.

10 Sukumar Ray's remarkable illustration of his Abol Tabol is a true complement to the nonsense verse. The text here makes a humorous reference to his illustration. 
In a poem like শব্দকল्প দ্রুম ('Sabdakalpa Droom'), which I call 'The Wordy Anarchy', there is no way one may convey the Bengali verbatim in English; for it is essentially a play with the sound of Bengali words and the fun is generated out of the inappropriateness of the sound and the sense. Sukanta Chaudhuri states that these are 'untranslatable' poems ('Sukumar Rayer Anubad,' Sukumar Parikrama, 147) and does not include them in his translation.

\section{শব্দকল্প দ্রুম (Sabdakalpa Droom) The Wordy Anarchy}

It banged and then it burst and how it boomed!

Not a cracker, only a flower just now bloomed!

It is a swoosh and a whoosh - a deluge primeval?

Don't panic, just the scent of the rose does travel!

Such a thump and what a thud! Oh, very scary?

But it's only the dew that drops, no need to worry.

Just hearken - what a splash and a smash and a pop!

Oh, only the moon sets - gob, and glob, drop, drop!

There is such a lot of rustle and bustle and tussle, The day dawns at last without so much of a hustle.

Then such a lot of buzzing along with chirping!

$\mathrm{O}$, it's only the ideas in my mind get churning.

You may listen to all those notes tra la la, hula la,

My mind is in a mood to dance at the gala.

With tinkle, tinkle, clank it gives such pain,

My heart breaks, it rumbles - truly, I do not feign.

There's such a noise, what a fright! Do they fight? Will they hit?

Do I sit? Or split? That's it. At once I quit.

\section{4}

One may wish to have a look at the stanzaic structures of the poems in Abol Tabol which are interestingly diverse. I have tried to follow Sukumar Ray's varied stanzaic structures. 
Sometimes even within the same poem I have used different line length, using a combination of short and long verse lines. In 'থুড়োর কল,' ('Khuror Kol'), 'Uncle's Device,' for example, the pentameter verse lines have been brought down to tetrameter ones at a point where the Uncle's engine gathers speed (see lines 21 to 26 in the poem below). The change is intended to capture this speed.

This is how I present Sukumar Ray's poem:

\section{খুড়োর কল (Khuror Kol) Uncle and his Wonder Engine}

The uncle of Chandidas has invented an engine, With it uncle's name wins fame in the whole regime.

As a babe this uncle showed early signs of mettle. He cried out, 'Gunga,' his first childhood prattle. Gagga, pappa, mamma're known childish babble, When a babe says, 'Gunga' it does indeed so baffle.

All said when he grew to be a man in his station, His brains would surely be a boon to the nation. The uncle's device now adds a zest to travel, You cannot but admire this outstanding marvel!

A distance that took you sev'n long hours, The uncle's machine in half the time covers!

The working of the device is straight and easy, I needed only five hours to learn it! There, see! This is all you have to do, and I give you a clue, Allow the device to attach itself to you, Then from the device you hang the choicest of goodies One loves to eat, and those that appeal to foodies, From luchies ${ }^{11}$ to cutlets, dhosas to pizzas to burgers, The mouth at once begins to water; the engine triggers.

${ }^{11}$ A thin and hollow, deep-fried round bread made of wheat-flour, a special favourite of all Bengalis. 
The more and more and more you run

To reach the goodies and have the fun,

The less and less and less may you

The goodies catch and eat them too.

But what you never did foresee,

You are soon there you wanted to be!

All admit the uncle's device is truly a marvel,

He's the greatest inventor that on earth did dwell.

In the last poem, আবোল-তাবোল ('Freakish Gibberish') the last four lines, unlike the preceding tetra meter lines, are written in pentameters. The pace is arrested here to convey the seriousness of the content of the poem that becomes heavy at this point. I cite the poem here:

\section{আবোল তাবোল (Abol Tabol) Freakish Gibberish}

In the cloudy expanse of the hazy night, In the sprinkled hue of the rainbow light, My mind at once does take a random flight.

I sing the song of my heart's delight. Today I'm free from all injunctions, And gone with the wind are all compunctions.

The sky is touched with tinge so pure

The air is charged with the magic lure, The fountain flows with exciting notes,

The dream-flower blooms in the wind and floats.

The eyes must catch the hue of the skies,

The mind, it's touched with the dye! It vies!

Today my dear before I bid goodbye

My mind and thoughts'll take off and fly.

I care not if it is meaningless.

I care not if it goes quite heedless.

My fancy from myself shall I set free,

To take its course in a whimsy spree.

My heart now hearkens to the sound 
Of drums, with that of thrill it's wound,

Tanta ratan ghatang ghach ${ }^{12}$

With words on words today I patch.

Now darkness is enfolded with light,

The bell-sound is heard with perfumed sight.

The secret envoy from dream-lands comes,

With the elements five I dance to the drums,

My partners are the greedy elephants

Quite upside down and I join their chants.

The queen of bees and the horse with wings!

The noisy brat to silence clings!

My bouquet is made of eggs from the mare's nest furled.

The dew-oozing moon now takes me to olden world.

At last my eyes are heavy with gentle sleep,

It is time for my song to recede in silence deep.

\section{5}

The sound management in the poems - the syllabic pattern, rhyme scheme and assonance and alliteration of Abol Tabolis also full of variety. And, as explained in Part I of this study, this aspect may pose serious difficulty for a translator. One might take for instance, 'কিম্তূত,' 'All in One': the longer lines contain 14 syllables, while the shortest ones have only 9 or 10 syllables; in between there are quite a few 11, 12 and 13 syllable lines. But whoever counts or notices these syllabic units in reading this masterpiece! Interestingly the shorter lines are composed of words which take the same time to articulate as the words contained in the long lines, thus creating the necessary rhythmic balance. Time substitutes for syllables.

${ }^{12}$ This acephalous nonsense verse line is taken verbatim from Bengali, which has no sense either, and its comic effect is in sharp contrast to the rather serious last lines of the poem. 
In this poem, 'কিষ্ভূত,' 'The Monstrosauraus' we find that in each couplet there is a midpoint alliteration and rhyme created regularly in each pair of lines throughout the thirty-six-line poem by the poet in addition to the very regular rhymed couplets, creating a music magic that sweeps over the irregular number of syllables in lines which range between 10 and 14 syllables! Thus here we have janoar (line 1, middle) matched by dhore tar (line 2 middle) while the rhymed ending of the two lines are as obvious - kimbhut and khutkhut.

Here is my version, with its sound patterns highlighted:

\section{কিষ্ভূত (Kimbhut) The Monstrosauraus}

For sure, this is the strangest of all the creatures, It grudges all it sees and all their features.

The whole day long it keeps on sulking and fretting, 'I'm all wrong,' thus it sighs in the fells and hills, regretting.

'I want this, I want that,' my needs're indeed so many,

They haunt and haunt! Alas! I can't choose any'The cuckoo has such a splendid singing voice, O giv'n a choice I'd change its voice with my noise. I envy the birds having fun as they race in the air, I must right now run and get me wings in a pair. The elephant's trunk so dangles all the way to the ground, Would anyone hear my grumbles if one for me I found. The kangaroo's secret's contained in its long hind legs, I wish I had obtained them, my god,' it begs.

'The Lion's-mane's the seat of its valour and its splendour, With mine I'll display my glamour, and cause such wonder. The iguana's tailpiece makes it a creature superior, I must get a designer tail for my posterior.' It keeps on sulking and nagging, fretting 'nd moaning. At last it gets all it was longing on one June morning. It sits quite still, ov'rcome with awe and thrill, Yet grave confusion, unwelcome, its mind does fill. 
'Would elephants prefer to hop and glide and fly?

Can Kangaroos on plantains manage to thrive?

Won't people jeer that all that cuckoo sound

They hear are made by a snub-nosed face that is round?

They'd scoff when they see a tubby old elephant flying,

They'd tug at my tail, and laugh and chase me booing,

"Hey, who goes there, quite nameless 'nd also homeless!"

This shows that I am now in a hopeless mess.

I'm not a horse or a frog, or a fly or a spider,

Nor am I a dog, a butterfly, or a tiger,

Nor am I a flea or an elephant, or a tree,

I'm not a bumble bee or an elk, I agree.

I can't be ev'n a poor old shoe or a wave,

Boo hoo! I'm just a nobody,' thus does it rave.

‘হুঁকোমুথো হ্যাংলা,' (Hookomukho Hyangla) 'Hookah-Faced Gluttony' likewise introduces sound jumble that nearly defies all translation. The poem has four-line stanzas. The mid-line sound is picked up by the last word in lines one and three: hyangla and bangla (line one); mane ki and jane ki - (line three). This pattern is thus used for lines one and three, while line 2 and line 4 have end rhymes (dekhecho and thekecho). And this intricate verse pattern is followed throughout. This sound scheme is so crucially important to the final effect of the poem that a translator cannot ignore it. Here is the translated version of the poem fully cited with its sound patterns highlighted:

\section{হকোমুখো হ্যাংলা (Hookohmukho Hyangla) Hookah-Faced Gluttony}

Hookah-Faced Gluttony Is a proper Bangali ${ }^{13}$.

Why does he look so dull and grim and grave?

13 'Bangali' would be the proper spelling of the local pronunciation of 'Bengali', a native of Bengal. 
The Art of Translation: On Translating Sukumar Ray's ...

Does anyone know the cause? Does anyone think and pause?

He was ever so cheerful, affable, gentle, and brave.

His uncle is Shyamadas The chief of opium storehouse,

He never had any other kith or kin,

Oh my poor little dearie

He looks so very teary,

So haggard and pale, so woebegone and thin.

With a hop and a bop, flip-flop, And a ramba and samba non-stop

He loved to waltz without pause all day so mirthful.

In a voice that's hoarse and husky He'd sing doh-re-mi ${ }^{14}$, ti-pi-pi.

He was so carefree, jolly and gleeful.

It was only at mid-day

we saw him all quite gay,

Relishing 'nd munching mashed banana raw.

Why then does he lie and sigh? Did the uncle suddenly die?

Or did he break his maw, or toe or his paw?

'Tut tut,' says Hookah-Face, 'It's truly a grim case, It's a fly-smacking and thwacking strategy.

It takes away my daylight, It keeps me awake all night,

If I solve it you may call me a giant prodigy.

If a fly does sit on my right,

Advises my foresight,

At once go smack it with the tail on your right;

If it sits on my left,

I am the least upset,

I know I can crush it with the tail on my left.

But what about the bugger That goes and sits at the centre?

I can't think which of my two poor tails do I try.

Just see what a hopeless impasse! How may I overcome the morass.

Alas! I have no more than two tails. Fie!'

Dissection is not the best approach to reading poems, we all know. Yet only a closer scrutiny can bring out the intricate process and planning that go into each creative work which,

${ }^{14}$ Indian notes are 'sa re ga ma'. 
after all, is not in a literal sense 'a spontaneous overflow of powerful feelings'. The source of fun and enjoyment in Sukumar Ray's Abol Tabol lies, among other things, also in its remarkable play with sound, which, for a translator or a critic, is an indispensible study; which, for a reader, is necessarily a process of enriching her experience and deepening her appreciation and admiration of the mastery of the poems. It is with this intention that $\mathrm{I}$ included here this closer reading of the intricacies of sound pattern in some poems of Sukumar Ray.

The veteran Bengali poet, Sankho Ghosh, analyses the intricate use of sound, metre and rhythm in some of the poems from Abol Tabol. He compares the earlier versions of these poems, which first came out in Sandesh, the children's magazine that the Ray family brought out in the early twentieth century, and the stringently revised versions that were prepared by Sukumar Ray from his deathbed for his Abol Tabol ('Atyukti O Atmanistha,' 'Over Statement and Dedication', Sukumar Parikrama, 15-22). This revision shows how austere were the standard of perfection that Sukumar Ray set himself.

\section{6}

In Part I of this essay it has already been pointed out that the falling rhythm of Trochee and Dactyls are more commonly found in Bengali, while English verse is prone to take to the rising rhythm such as Iambus and Anapest (also mentioned by Sukanta Chaudhuri, (Sukumar Parikrama, 144). In translating Sukumar Ray in English a basic Iambic pattern rather than the falling rhythm of trochee and dactyl would appear more natural. Among the very few exceptions I allowed myself in using the falling rhythm, I wish to cite 'বাবুরাম সাপুড়ে,' 'Baburam the Snakeman': 


\section{বাবুরাম সাপুড়ে Baburam the Snakeman}

Baburam, snakeman, whither are you bound?

Get me please two snakes all safe 'n' sound.

Snakes those're tame and also quite sane,

Feed on milk rice and are limp 'n' lame.

Those that sting not, spring not, bite or spite,

Those which're truly ashamed to fright ' $n$ ' fight,

Bring only Snakes those're stingless, toothless,

Hornless, clawless, hoodless, harmless,

Snakes that hiss not, fizz not're blind 'n' kind,

Race not, run not, please go and find.

I will catch them, teach them, preach them too!

Box them, bash them, beat them black 'nd blue.

Finally, in this last section of the essay, we take a quick glimpse into one aspect of the complications raised by the process of translating the content of Sukumar Ray's Abol Tabol in English. Our observation will include only a brief sample of what could be a book length discussion, for expectedly, the process involves complications of a widely different nature. Along with its stylistic features the subject matter of the nonsense verse necessarily poses a serious challenge to the translator. Nonsense is generated through not only how it is said, but also what is being said. And translating what makes sense is far easier than what goes to make nonsense.

The fun evoked in the nonsense world of Sukumar Ray is inextricably embedded in the eccentricity of the Bengali lingual practices. The 'nonsense' is generated often by using and sometimes twisting the sense in which ideas, words and phrases and sayings are familiarly used in the Bengali lingual corpus. We shall cite two instances from Abol Tabol. 
In Sukumar Ray's poem, গোঁফ চুরি (Gomph Churi) - 'The Stolen Moustache' the boss at the head office is said to be shot with a spell of uncontrollable anger when the clerks in his office try to persuade him, contrary to his notion, that his moustache is not stolen. To describe the angry boss, Sukumar Ray, uses a typical expression popular in local parlance: that of using the image of frying the eggplant in a pan full of hot oil রেগে আগুন তেলে বেগুন - 'rege aagun, tele begoon'. This association of one's expression of anger and the actual act of frying the vegetable called the বেগুন (begoon) or eggplant is typically born out of a Bengali socio-cultural context. The behaviour of an angry person and that person's flushed angry face are described in terms of ideas that take one to a typically favourite culinary practice of Bengali cuisine and not without some humour in it. And this association is not nonsense per se, as it is already available in the current usage, and Sukumar Ray has not coined this expression. At page 1058 of the Bangiya Sabdakosh, Vol. 1, we find an entry for this expression, which was already in currency when Sukumar Ray used it. This association between the two unrelated acts, therefore, need not have to be decoded to a native of Bengal. But it needs an absurdly elaborate explanation in any other language. These are indeed untranslatable eccentricities of Bengali speech act, a product of Bengali socio-cultural context, no less typical than any other alien context which surely generates its own set of such elements.

We may take such another expression typical in its Bengali association and simply not found and therefore cannot be translated in English. The poem entitled নেড়া বেলতলায় यায় কবার - Neda Baeltolay Jaay Kobaar - is a nonsensical and literal extension of a Bengali saying which really means 'once bitten twice shy'; but it is rooted in the comical notion that when a man with a shaven pate is struck with a 'bael' a fruit 
(also known as wood apple or marmelos) that has a very hard outer rind and can badly damage if it falls on one's head. A ripe bael often drops from its stem with shell and all. The popular saying tells us that a man who is hurt by the bael when it drops on his head from the tree is cautious not to go beneath the tree a second time. This very explanation shows how absurdly preposterous and unnecessary elaboration of the saying can be; only the enterprising King in the poem is frustrated that no wise man has ever specified as to the number of times a clean shaven man may go beneath a 'bael' tree. The nonsense, therefore, is not in the saying, which is a Bengali proverb, but in the ridiculous act of the King in exploring the entire retinue of all his available human resources to find an answer to the query.

\section{8}

Translation of Abol Tabol can indeed be termed as a daunting task. And, therefore, we may also claim that Abol Tabol is one of those extraordinary books which any translator would be proud to have successfully translated. The honour of the first ever attempt of translating some of Sukumar Ray's poems from Abol Tabol naturally belongs to his celebrated son, Satyajit Ray, who translated ten poems from Abol Tabol which was published in 1970. After Satyajit Ray broke the ice with his 10 poems there have been quite a few good translations, which are mentioned in the books cited section of the this essay. But the work continues, for more and more people are taking interest in Sukumar Ray studies; it is only expected that more and more translations of the great master will be written in English and other Indian and non-Indian languages. It would 
be a matter of regret indeed if Sukumar Ray's Abol Tabol, remained confined to the Bengali readers ${ }^{15}$.

\section{References}

AyUB, ABU SAID. 1976. Galiber Gazal Theke, (From Galib's Gazals). Kolkata: Dey's Publishing.

BANDYOPADHYAY, HARICHARAN. 1966. Bangiya Sabdakosh:

A Bengali-Bengali Lexicon. Vol. 1. 2016. Sahitya Akademi: New Delhi.

Barnstone, Willis. 1993. The Poetics of Translation. History, Theory, Practice. London:Yale University Press.

ChatTARJ, SAMPURNA. 2004. Wordygurdyboom!(Abol Tabol).

The Nonsense World of Sukumar Ray. Gurugram: Penguin Random House India.

Chaudhuri, SuKanta. 1989. Sukumar Rayer Anubad, ('Translation of Sukumar Ray'), Sukumar Parikrama. In Pabitra Sarkar (ed.), A Collection Essays on Sukumar Ray. Calcutta:Paschim Banga Bangla Academy.

CHAUdHURI, SUKANTA. 1987. The Select Nonsense of Sukumar Ray. New Delhi: OUP.

ELIOT, T, S. 1976. The Sacred Wood. Essays on Poetry and Criticism. B. I. Publications: New Delhi.

GHosh, SANKHA. 1989. Atyukti O Atmanistha, ('Over Statement and Dedication'), Sukumar Parikrama. In Pabitra Sarkar 9ed.), A Collection Essays on Sukumar Ray. Calcutta: Paschim Banga Bangla Academy.

GuPTA, Nirmal. 1988. Whymsy Wave. Nonsense World of Sukumar Ray. Prakash Bhavan: Calcutta.

\footnotetext{
${ }^{15}$ In 2023, we are going to see the death centenary of the great master. We can only expect that new works, including translations, will appear to mark the occasion in a befitting manner. All Bengali quotations by scholars and critics have been translated from the original by the writer.
} 
The Art of Translation: On Translating Sukumar Ray's ...

LEAR, EDWARD. 1846. There was an Old Man with an Owl, Two Hundred Years of Nonsense. The Works of Edward Lear. http://lear200.com/there-was-old-man-owl.

McGowan, Kate. 2006. Structuralism and Semiotics. In Simon Malpas and Paul Wake (eds.), The Routledge Companion to Critical Theory. London: Routledge.

PARrotT, E. O. (ed.). 1983. The Penguin Book of Limericks. 1991. London: Bloomsbury Books.

RAY, SATYAJIT. 1986. Torai Bandha Ghorar Dim (A Bouquet of Eggs from the Horses's Nest). Calcutta: Ananda Publishers Private Limited.

Roy, NiladRI. 2017. Rhymes of Whimsy. The Complete Abol Tabol by Sukumar Ray. California: Haton Cross Press.

SAMPSON, FIONA. 2012. Creative Translation. In David Morleyand and Philip Neilsen (eds.), The Cambridge Companion to Creative Writing. Cambridge: Cambridge University Press.

Shelley, P. B. 1909-14. A Defence of Poetry and Other Essays. English Essays: Sidney to Macaulay. The Harvard Classics. https://edisciplinas.usp.br/mod/resource/view. php?id=2573689.

Woodings, R. B. (ed.). 1968. Shelley. Macmillan Education. Modern Judgments Series. In P.N. Furbank (ed.), Selection of Critical Essays. London: Macmillan and Co Ltd.

$* * *$

\section{Cite this Work}

SRInIVASAN, Uma RaY. 2020. The Art of Translation: On Translating Sukumar Ray's Abol Tabol in English. Translation Today, Vol. 14(2). 81105. DOI:10.46623/tt/2020.14.2.ar5 